\title{
Efeito do Uso de Anti-retrovirais sobre o Metabolismo Glicídico de Gestantes Portadoras do HIV-1 e Parâmetros Antropométricos e Bioquímicos do Neonato
}

\author{
Effect of Antiretroviral Agents Use on Glucide Metabolism in HIV-1 Infected Pregnant Women \\ and on Anthropometric and Biochemical Parameters of Newborn Infant
}

\author{
Autora: Patrícia El Beitune \\ Orientador: Prof. Dr. Geraldo Duarte
}

Tese de Doutorado apresentada ao Curso de Pós-Graduação da Faculdade de Medicina de Ribeirão Preto da Universidade de São Paulo para obtenção do Título de Doutor em Medicina; Área de concentração em Tocoginecologia, em 3 de Junho de 2004.

Objetivos: estudar o efeito dos anti-retrovirais em gestantes portadoras do HIV-1 sobre o metabolismo glicídico materno e parâmetros antropométricos e bioquímicos do neonato.

Pacientes e Métodos: estudo prospectivo com 57 gestantes divididas em três grupos. O Grupo AZT foi composto de 20 gestantes infectadas pelo HIV-1 que preenchiam os requisitos para uso profilático do AZT $(\mathrm{CD} 4>500$ células $/ \mathrm{ml}$ ). O Grupo TT foi composto de outras 25 gestantes com indicação clínica e/ou laboratorial para receberem terapia anti-retroviral triplice $(\mathrm{AZT}+3 \mathrm{TC}+\mathrm{NFV})$. O Grupo Controle foi constituído de 12 gestantes consideradas normais do ponto de vista clínico e laboratorial. As amostras sangüíneas para dosagens plasmáticas de glicemia em jejum, insulina sérica em jejum, área sob a curva das glicemias e insulinas após o teste oral de tolerância à glicose com 75 g (TOTG-75 g) foram obtidas em quatro períodos durante a gravidez, em intervalos equidistantes de tempo. O prognóstico perinatal levou em consideração as taxas de prematuridade, peso ao nascimento, restrição de crescimento intra-uterino, óbito fetal e mortalidade neonatal. Adicionalmente, avaliou-se com o sangue do cordão umbilical do recém-nascido (RN) a gasometria da artéria umbilical, a insulina, os valores da hemoglobina, transaminases hepáticas e amilase. A análise estatística foi realizada utilizando-se testes não paramétricos do qui-quadrado, Mann-Whitney, Friedman e KruskalWallis com o teste post-hoc de Dunn, considerando p < 0,05 como nivel de significância.
Resultados: observou-se homogeneidade entre os dados demográficos e antropométricos de realce e, excluidas gestantes com história pessoal e familiar de diabetes mellitus, houve associação entre uso de inibidores da protease com o desenvolvimento de intolerância à glicose durante a gestação, no período entre 33-38 semanas. Os valores da mediana da área sob a curva das dosagens das glicemias, durante 120 minutos, foi 11 $685 \mathrm{mg} / \mathrm{dL}-2 \mathrm{~h}$ para o grupo controle, $13477 \mathrm{mg} / \mathrm{dL}-2 \mathrm{~h}$ para o grupo AZT e $13650 \mathrm{mg} / \mathrm{dL}-2 \mathrm{~h}$ para o grupo TT $(p=0,049)$. As gestantes portadoras do HIV-1 em uso de anti-retrovirais, durante a gestação, apresentaram diferença significativa da área sob a curva da insulina e dos níveis séricos de insulina neonatal ao nascimento em relação ao grupo controle. Houve redução dos níveis de hemoglobina ao nascimento de RN do grupo TT ( $\mathrm{p}<$ 0,05). Não houve diferença entre os grupos em relação à duração da gestação, indices de Apgar, classificação antropométrica neonatal, transaminases hepáticas, bilirrubinas, amilase, plaquetas e contagem de linfócitos absolutos do RN. Não houve nenhum caso de transmissão vertical do HIV-1.

Conclusões: verificou-se associação entre uso de inibidores da protease com o desenvolvimento de intolerância glicêmica durante a gestação e menores níveis séricos de insulina e hemoglobina neonatal.

Palavras-chave: Infecções na gravidez. AIDS. Prognóstico perinatal. Metabolismo glicídico.



RBG0-v.26, $n^{0} 7,2004$ 